\title{
Plasma Electrolytic Oxidation of Titanium Implant Surfaces: Microgroove-Structures Improve Cellular Adhesion and Viability
}

\author{
PHILIP HARTJEN $^{1 *}$, ALEXIA HOFFMANN ${ }^{1 *}$, ANDERS HENNINGSEN ${ }^{1}$, MIKE BARBECK $^{1}$, \\ ALEXANDER KOPP ${ }^{2}$, LAN KLUWE ${ }^{1}$, CLARISSA PRECHT ${ }^{1}$, OLIVIA QUATELA ${ }^{1}$, \\ ROBERT GAUDIN $^{3}$, MAX HEILAND ${ }^{3}$, REINHARD E. FRIEDRICH ${ }^{1}$, \\ CHRISTIAN KNIPFER $^{1}$, DANIEL GRUBEANU ${ }^{4}$, RALF SMEETS ${ }^{1}$ and OLE JUNG ${ }^{1}$ \\ ${ }^{1}$ Department of Oral and Maxillofacial Surgery, \\ University Medical Center Hamburg-Eppendorf, Hamburg, Germany; \\ ${ }^{2}$ Meotec GmbH \& Co. KG, Aachen, Germany; \\ ${ }^{3}$ Department of Oral \& Maxillofacial Surgery, Charité - Universitätsmedizin Berlin, Berlin, Germany; \\ ${ }^{4}$ Fresenius University Medical School, Idstein, Germany
}

\begin{abstract}
Background/Aim: Plasma electrolytic oxidation $(P E O)$ is an established electrochemical treatment technique that can be used for surface modifications of metal implants. In this study we to treated titanium implants with PEO, to examine the resulting microstructure and to characterize adhesion and viability of cells on the treated surfaces. Our aim was to identify an optimal surface-modification for titanium implants in order to improve soft-tissue integration. Materials and Methods: Three surface-variants were generated on titanium alloy Ti6Al4V by PEO-treatment. The elemental composition and the microstructures of the surfaces were characterized using energy dispersive $X$-ray spectroscopy, scanning electron microscopy and profilometry. In vitro cytocompatibility of the surfaces was assessed by seeding L929 fibroblasts onto them and measuring the adhesion, viability and cytotoxicity of cells by means of live/dead staining, XTT assay and LDH assay. Results: Electron microscopy and profilometry revealed that the PEOsurface variants differed largely in microstructureltopography, porosity and roughness from the untreated control material as
\end{abstract}

This article is freely accessible online.

*These Authors contributed equally to this study.

Correspondence to: Dr. Ole Jung, Department of Oral and Maxillofacial Surgery, University Medical Center HamburgEppendorf, Martinistrasse 52, 20246 Hamburg, Germany. Tel: +49 0407410 53259, e-mail: ol.jung@uke.de

Key Words: Plasma electrolytic oxidation (PEO), in vitro cytocompatibility, surface topography, cell adhesion. well as from one another. Roughness was generally increased after PEO-treatment. In vitro, PEO-treatment led to improved cellular adhesion and viability of cells accompanied by decreased cytotoxicity. Conclusion: PEO-treatment provides a promising strategy to improve the integration of titanium implants with surrounding tissues.

Titanium and its alloys have been the metals of choice as dental implant materials due to their fulfilling the required criteria of having a low density, acquiring high mechanical properties, corrosion resistance, as well as offering acceptable biocompatibility (1-4). Nevertheless, there is still room for improvement, particularly in terms of surface characteristics. This is due to the fact that the outer implant surface is the interface that elicits the host response and determines how the immediate microenvironment of cells and tissues in the vicinity of the implant will respond $(3,5)$. Introducing microstructure and roughness onto the surface, or/and coating the surface with other materials may provide a feasible approach to improve the tissue-integration of titanium implants (4-6).

Different treatments have been employed to alter the surface characteristics of implants. Some of these can be classified as physical treatment modalities, such as conventional sandblasting and hydrothermal treatment, while others fall into the category of chemical treatment, such as pickling, chemical vapor deposition, hydroxyapatite coatings through plasma spraying, and anodic oxidation $(1,7,8)$. However, surface features (e.g. hardness, corrosion resistance, bonding strength of the surface layer) using the aforementioned techniques were not satisfactory. Regarding cellular response, none of the surface treatment techniques can be considered superior $(5,8)$. 
Table I. PEO-treatment and summary of features of treatment surfaces.

\begin{tabular}{|c|c|c|c|c|}
\hline & Untreated & PEO1 & PEO2 & PEO3 \\
\hline \multicolumn{5}{|c|}{ Electrolyte ingredients present during PEO treatment } \\
\hline$\left(\mathrm{PO}_{4}\right)^{3-}$ & & + & + & + \\
\hline$\left(\mathrm{NH}_{4}\right)^{+}$ & & + & + & + \\
\hline $\mathrm{OH}^{-}$ & & + & + & + \\
\hline $\mathrm{F}^{-}$ & & + & + & \\
\hline$\left(\mathrm{BO}_{3}\right)^{3-}$ & & + & + & \\
\hline $\mathrm{Na}^{+}$ & & & + & + \\
\hline $\mathrm{Ca}^{2+}$ & & & & + \\
\hline \multicolumn{5}{|l|}{ Roughness (Sa) } \\
\hline & 1.42 & 0.64 & 2.17 & 1.52 \\
\hline \multicolumn{5}{|l|}{ Microstructure } \\
\hline Flat & + & + & & \\
\hline Porous & & + & + & + \\
\hline Grooves & & & + & + \\
\hline protrusions & & & + & + \\
\hline Description & $\begin{array}{c}\text { Flat, no grooves, } \\
\text { no protrusions }\end{array}$ & $\begin{array}{c}\text { Flat, small round } \mathrm{g} \\
\text { rooves }(<1 \mu \mathrm{m} \text { diameter }) \\
\text { no protrusions }\end{array}$ & $\begin{array}{l}\text { Porous, occasional round and } \\
\text { drop-shaped grooves }(1-10 \mu \mathrm{m} \\
\text { diameter), protrusions } \\
\quad(5-10 \mu \mathrm{m} \text { diameter })\end{array}$ & $\begin{array}{l}\text { Porous, drop-shaped } \\
\text { grooves }(5-15 \mu \mathrm{m}) \text {, } \\
\text { protrusions }(5-15 \mu \mathrm{m} \\
\text { length, } 5 \mu \mathrm{m} \text { width })\end{array}$ \\
\hline \multicolumn{5}{|l|}{ In vitro features } \\
\hline Morphology & Round & $\mathrm{Bi}-$ and poly-polar & $\mathrm{Bi}$ - and poly-polar & $\mathrm{Bi}$ - and poly-polar \\
\hline Viability (XTT-assay) & $57 \%$ & $63 \%$ & $82 \%$ & $82 \%$ \\
\hline Difference to untreated & & Not significant, $p=0.8125$ & Not significant, $p=0.0625$ & Not significant, $p=0.1875$ \\
\hline Cytotoxicity (LDH-assay) & $169 \%$ & $147 \%$ & $129 \%$ & $126 \%$ \\
\hline Difference to untreated & & Not significant, $p=0.4375$ & Not significant, $p=0.2500$ & Not significant, $p=0.1250$ \\
\hline
\end{tabular}

Plasma electrolytic oxidation (PEO) consists of an electrochemical treatment resulting in the production of a more stable ceramic oxide layer in comparison with anodic oxidation $(6,9-12)$. As the name suggests, the PEO process is based on a local oxidation of a metal substrate. Such oxidation takes place in an electrolytic bath by means of a high potential provided between the sample and the counter electrode. After a potential buildup phase, the system reaches its energetic minimum by displaying a surface distribution of characteristic local plasma discharges. The resulting plasma discharges are considered as a stabilizing factor of the oxide layer, where they modify it into a ceramic-like structure (12). This ceramic layer revealed superior characteristics in terms of stability, mechanical strength, bonding strength, corrosion and wear resistance $(3,5,8)$. Simultaneously, PEO-treatment also improved the cellular host reaction in regard to cell adhesion, proliferation, and osteoblastic differentiation as reported in both in vitro and in vivo studies $(1,2,5,8,10,12)$.

By changing parameters (e.g. applied potential, time, temperature, electrolytic composition), PEO can offer a vast variability of surface topographies (e.g. individually tailored for specific cell entities) and compositions $(1,6)$. However, these surfaces have not been evaluated for their effect on living cells.
In this study, by further varying the PEO process parameters and electrolyte composition, three different types of porous, grooved and drop-shaped Ti6Al4V PEO-surfaces were generated. We characterized the physical features of these surfaces and evaluated their biocompatibility in terms of cell adhesion as well as the viability of cells cultured directly on these surfaces.

\section{Materials and Methods}

Specimen preparation. One mm thick titanium discs were cut from an $18 \mathrm{~mm} \varnothing$ rod of commercially available grade 5 Ti6Al4V (alpha/beta-alloy) using electrical discharge machining (Sodick AQ537L, Duesseldorf, Germany). After polishing with silicon carbide paper (600 grit), the specimens were washed in ethanol for $10 \mathrm{~min}$ and in $15 \% \mathrm{HF} / 30 \% \mathrm{HNO}_{3}$ for $15 \mathrm{~s}$ in order to remove oxide layers resulting from the cutting and polishing process. Finally, all samples were washed with distilled water for $15 \mathrm{~min}$ in an ultrasonic bath and dried on a sterile blanket at room temperature.

Plasma electrolytic oxidation. Three different PEO-surfaces were generated using distinctive combinations of electrolyte-components (Table I). PEO was achieved using a pulsed rectifier set (Meotec MPEO A1, Aachen, Germany). Positive and negative pulsed galvanostatic currents of $2 \mathrm{~A}$ and $3 \mathrm{~A}$ with voltages from $0 \mathrm{~V}$ to 
A


PEO-1


PEO-2

PEO-3

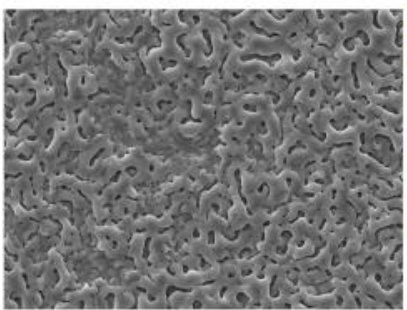

B
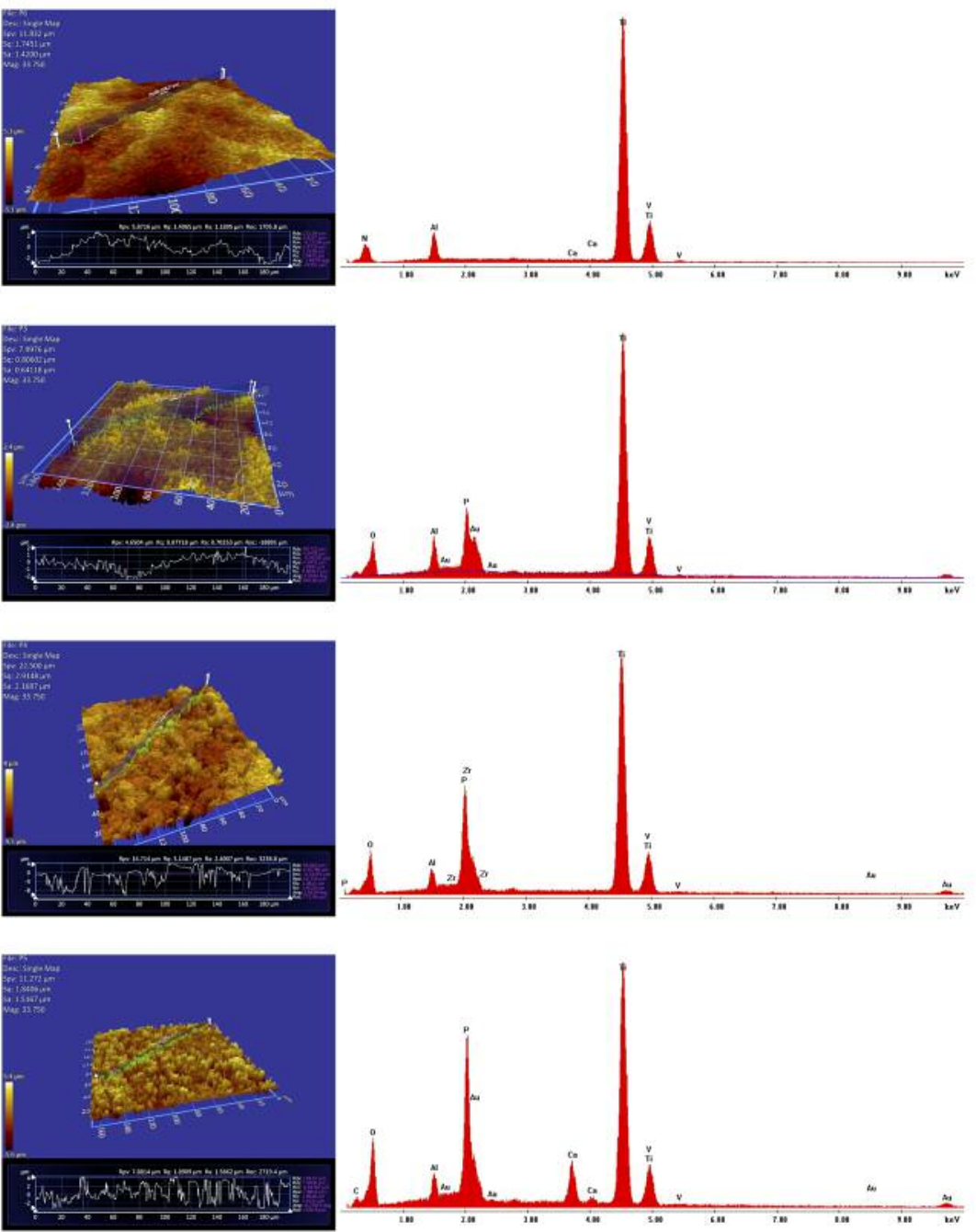

Figure 1. Physical features of PEO-surfaces. A) Scanning electron microscopy with resolution of $10 \mu \mathrm{m}$; B) profilometry; C) energy-dispersive Xray spectroscopy.

$500 \mathrm{~V}$ were applied using two feed cables. The electrochemical cell consisted of a titanium specimen as anode and platinum mesh as cathode. Pulse frequency was set to $20 \mathrm{~Hz}$ and discs were treated for up to $15 \mathrm{~min}$. After processing, all samples were rinsed with distilled water in an ultrasonic bath for $15 \mathrm{~min}$ and dried on a sterile blanket at room temperature.

Physical and chemical characterization of the surfaces. The coated surfaces were imaged by scanning electron microscopy (Philips XL30 CP, Amsterdam, Netherlands) and energy-dispersive X-ray spectroscopy. 3D structures and roughness of coated surfaces were characterized using a non-contact optical 3D profilometer (ZygoLOT ZeGage, AMETEK GmbH, Wiesbaden, Germany).

In vitro characterization of the surfaces. Cells, assays and other in vitro settings were applied as described in our previous work (13), except that the assay volume was $1 \mathrm{ml}$. L929 mouse fibroblasts were used for all assays as recommended in EN ISO 10993-5/-12 for cytocompatibility testing.

Untreated titanium discs were used as references for comparison. For negative controls, cells were directly seeded onto the culture surface of 12-well plates. The absorbance of blank controls (without cells) was subtracted from the resulting signal.

Data evaluation. The mean absorbance of the blank controls (cell culture medium without cells) was calculated and used as the baseline. After subtracting the baseline, mean absorbance and standard deviation were calculated from the corresponding replicates for each test material or control. The mean absorbance of test materials was normalized against that of controls (cells grown on cultural surface of the 12-plate without titanium discs). 


\section{Untreated}

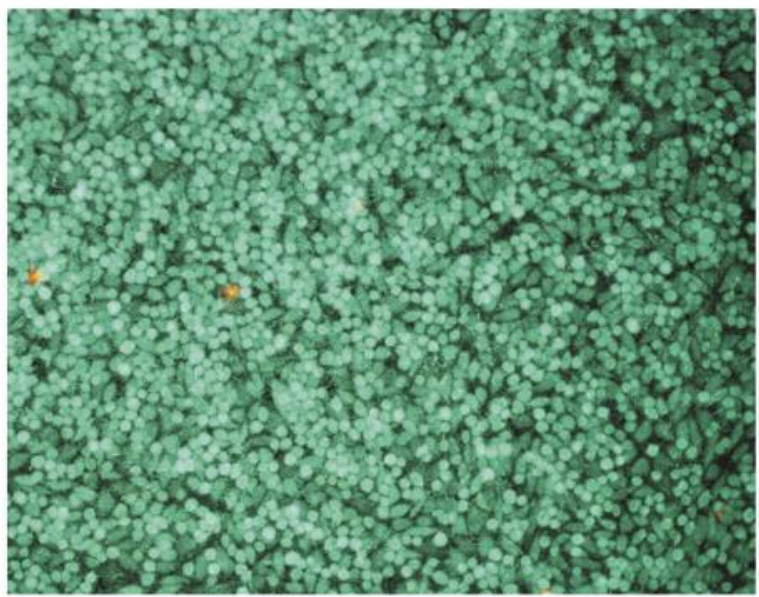

\section{PEO-2}

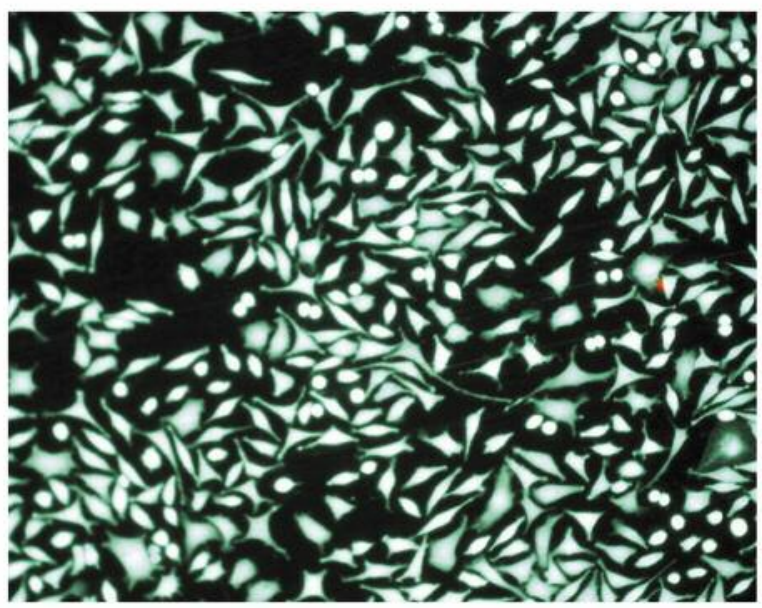

PEO-1



PEO-3

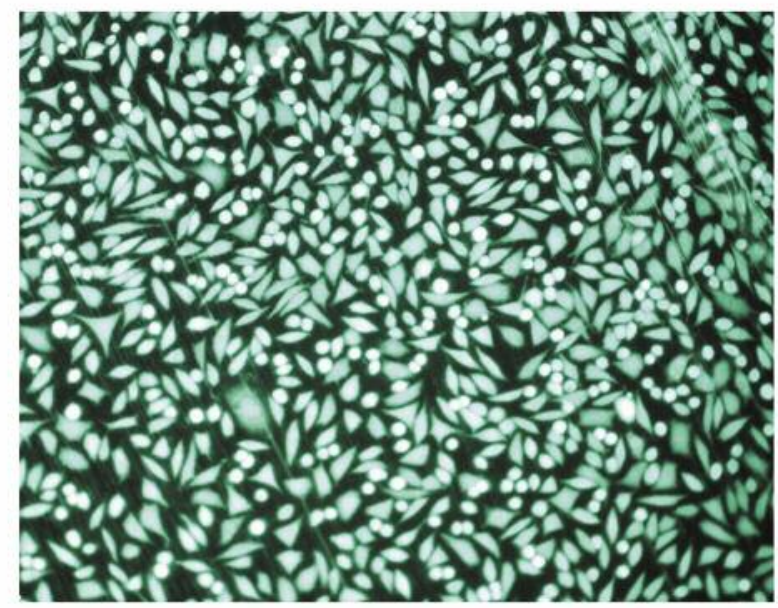

Figure 2. Direct live-dead staining of cells on the PEO-surfaces. Living cells are FDA positive and therefore green. Dead cells are stained by red PI in their nuclei.

Statistical analysis. Statistical analysis was carried out using the software GraphPad Prism 5.0 (GraphPad Software, San Diego, CA, USA). All groups were tested for normal distribution with the Kolmogorov-Smirnov test. The differences between the untreated samples and the PEO-treated samples were compared using the Wilcoxon matched-pairs signed rank test because some groups were not normally distributed. The test hypothesis was always two-tailed and $p$-values $<0.0125$ (Bonferroni correction) were considered to be statistically significant.

\section{Results}

Physical and chemical characterization. As expected, titanium, aluminum and vanadium were detected in the untreated as well as all PEO-treated surfaces as evidenced by corresponding peaks in the energy dispersive X-ray spectra.
Large amounts of phosphate were detected in PEO-1 and PEO-3. Calcium was detected only in PEO-3 (Figure 1).

The microstructures of the three PEO-treated titanium surfaces differed from each other and from the untreated surface (Figure 1, Table I). PEO-1 was flat, with roughness even below that of the untreated titanium surface. PEO-2 and PEO-3 showed increased roughness. All three PEO-treated surfaces were characterized by the presence of various grooves and protrusions differing in shape and size (Figure 1, Table I).

In vitro characterization. Regardless of the PEO treatment, large numbers of green fluorescein diacetate (FDA) positive vital cells and only sparse red propidium iodide (PI) positive dead cells were visible on all surfaces (Figure 2). However, 

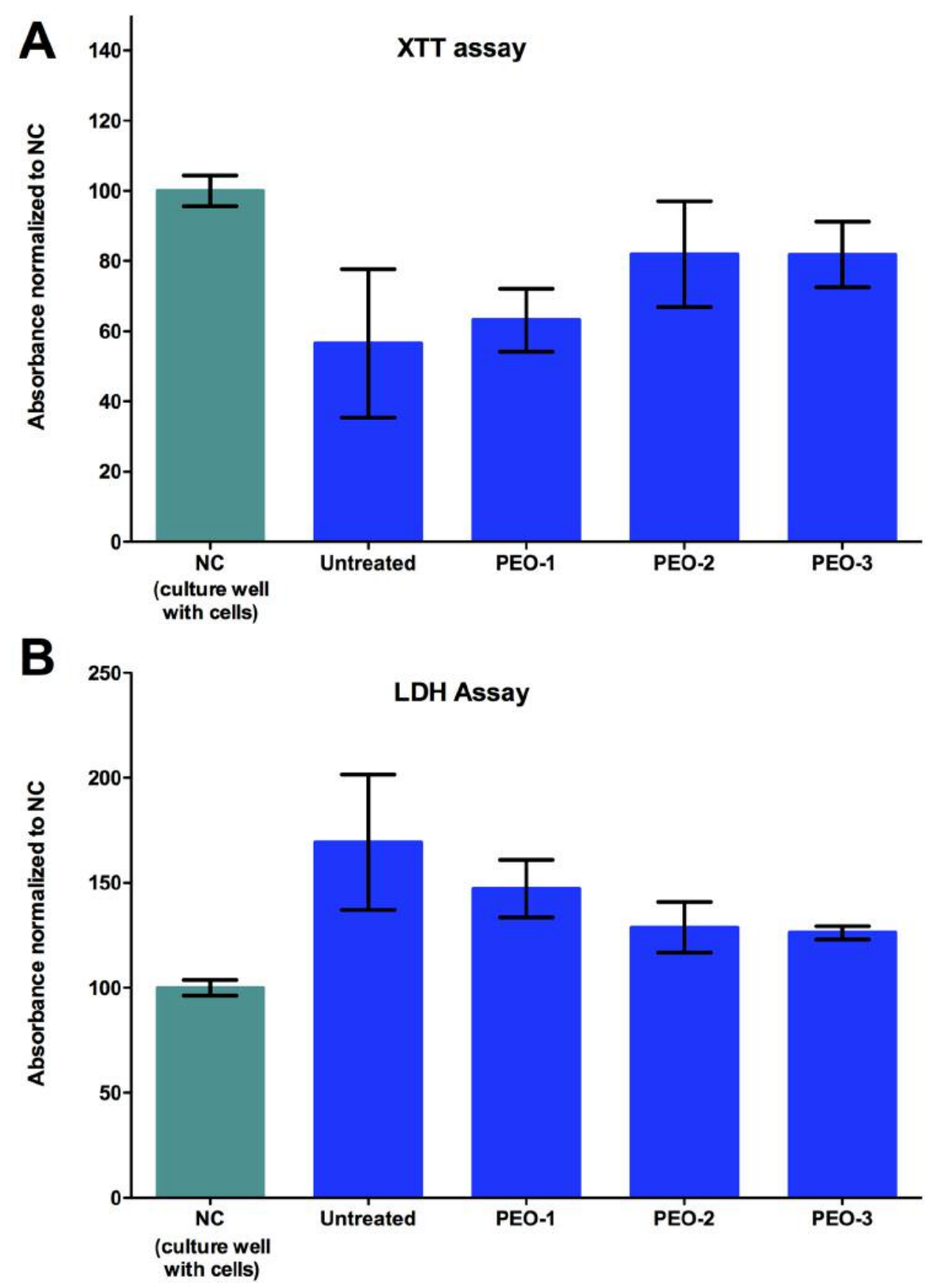

Figure 3. Viability (A) and cytotoxicity (B) of cells cultured on PEO-treated surfaces. Absorbance values for cells cultured on standard cultural surfaces were taken as negative control and set to $100 \%$. Values for cells cultured on treated (PEO-1, -2, -3) and untreated titanium surfaces were normalized against the negative controls.

the cells attached to the untreated titanium surface exhibited a round morphology, indicating poor adhesion. By contrast, spindle-shaped morphologies were observed for the majority of cells on the three PEO-treated surfaces, suggesting substantially improved adhesion.
In concordance, higher viability and lower cytotoxicity (Figure 3A and B respectively and Table I) were measured for cells on the PEO-treated surfaces than for cells on the untreated titanium surfaces. However, the differences were not statistically significant for both XTT- and the LDH-assays. 


\section{Discussion}

Our results demonstrate that with PEO technology, various microstructures can be introduced onto the surfaces of titanium implants by using a variety of electrolytes and electrical parameters. None of the PEO-treated samples showed increased cytotoxicity, suggesting that the electrolytes either were retained within the microstructure, or were released without being toxic.

In fact, PEO-treatment reduced cytotoxicity and increased viability of cells cultured directly on the surfaces. The spindle-shaped morphology of the cells attached to the PEOtreated surfaces provide sufficient evidence for an improved cell adhesion. Though cells also attached to the untreated titanium surface, most of them showed a round morphology, indicating insufficient adhesion. The improved adhesion and cell viability are likely due to the introduced microstructures of the surfaces and not merely the result of increased roughness, as one PEO-surface (PEO-1) was less rough than the untreated surface but had small round grooves. This finding might be possibly supported by a recent study, which concluded that having grooves as microstructures resulted in a higher shear strength than porous morphology due to enhanced interlocking of the adhesive into the grooved surface (1). In the same manner, cells in our study might have found it easier to interlock and grasp within the grooves.

Furthermore, in line with the enhanced cell attachment presented in our results for the porous PEO-2 and 3 surfaces, a previous study introduced a porous layer by PEO treatment (14). The resulting surface offered specific cues to guide the osteoblasts in a phenomenon referred to as contact guidance phenomenon, concluding a confirmatory increase in cell adhesion potential through what might be mechanical interlocking brought about by porosity (3). Moreover, Whiteside et al. reported more specific criteria for biologically advantageous porosity, where surfaces with a greater number of finer pores are favored for cell attachment (8). Another factor found to help with cellular recruitment, particularly with the targeted osteoblast cells, is the incorporation of calcium and phosphate ions within the oxide layer. These incorporated ions promote osteoinduction by causing initial adhesion of osteoblast ligands $(6,12)$. One generated surface in our study (PEO-3) showed the combined presence of incorporated calcium and phosphate ions, holding potential for osteoinduction.

Although the in vitro features of the PEO-treated materials were clearly improved compared to the untreated titanium surface, they did not yet reach the level of the standard cultural surface in the cell culture plates regarding both viability and cytotoxicity. Therefore, there is still room for further improvement. Since large numbers of various structures can be generated by varying electrolytes and electrical PEO parameters, we are confident to find better PEO-treatment conditions for optimal tissue integration of titanium implants in the future.

In the present study, only three PEO surfaces were evaluated. It is, therefore, too early to address the issue of possible correlations between microstructural features, cell adhesion and viability. However, as we screen more variants of PEO-surfaces, we expect to gain insight into the correlation between micro-topography and cellular attachment. Future studies should also include other types of cells in vitro, especially those of osteoblastic origin. Furthermore, biocompatibility and tissue integration of PEOcoated surfaces need to be assessed in vivo. Finally, mechanical features and corrosion resistance of the PEOtreated surface should be investigated.

\section{Conflicts of Interest}

AK is employed by the Meotec GmbH \& Co. KG, a company that is specialized in electrochemical surface treatment of metals. Meotec manufactured the test materials and performed the PEOcoating technology in this study.

\section{Acknowledgements}

This study was funded by the Bundesministerium für Bildung und Forschung, BMBF (FKZ 0316206A-C, BIOMAGIK). This work is the result of the collaboration within the BIOMAGIK consortium (University Medical Center Hamburg-Eppendorf and Meotec GmbH \& Co. KG Aachen, Germany).

\section{References}

1 Galvis OA, Quintero D, Castaño JG, Liu H, Thompson GE, Skeldon P and Echeverría F: Formation of grooved and porous coatings on titanium by plasma electrolytic oxidation in h2so4/h3po4 electrolytes and effects of coating morphology on adhesive bonding. Surface and Coatings Technology 269(Suppl C): 238-249, 2015.

2 Kazek-Kesik A, Kuna K, Dec W, Widziolek M, Tylko G, Osyczka AM and Simka W: In vitro bioactivity investigations of ti-15mo alloy after electrochemical surface modification. J Biomed Mater Res B Appl Biomater 104(5): 903-913, 2016.

3 Wu J, Liu ZM, Zhao XH, Gao Y, Hu J and Gao B: Improved biological performance of microarc-oxidized low-modulus ti24nb-4zr-7.9sn alloy. J Biomed Mater Res B Appl Biomater 92(2): 298-306, 2010.

4 Li Z, Yi J, Huang B, Wu X, Qiao W, Luo X and Chen Z: Ultraviolet irradiation enhanced bioactivity and biological response of mesenchymal stem cells on micro-arc oxidized titanium surfaces. Dent Mater J 34(2): 135-147, 2015.

5 Chung CJ, Su RT, Chu HJ, Chen HT, Tsou HK and He JL: Plasma electrolytic oxidation of titanium and improvement in osseointegration. J Biomed Mater Res B Appl Biomater 101(6): 1023-1030, 2013.

6 Santiago-Medina P, Sundaram PA and Diffoot-Carlo N: The effects of micro arc oxidation of gamma titanium aluminide surfaces on osteoblast adhesion and differentiation. J Mater Sci Mater Med 25(6): 1577-1587, 2014. 
7 Kazek-Kesik A, Krok-Borkowicz M, Pamula E and Simka W: Electrochemical and biological characterization of coatings formed on ti-15mo alloy by plasma electrolytic oxidation. Mater Sci Eng C Mater Biol Appl 43: 172-181, 2014.

8 Whiteside P, Matykina E, Gough JE, Skeldon P and Thompson GE: In vitro evaluation of cell proliferation and collagen synthesis on titanium following plasma electrolytic oxidation. $\mathrm{J}$ Biomed Mater Res A 94(1): 38-46, 2010.

9 Farraro KF, Kim KE, Woo SL, Flowers JR and McCullough MB: Revolutionizing orthopaedic biomaterials: The potential of biodegradable and bioresorbable magnesium-based materials for functional tissue engineering. J Biomech 47(9): 1979-1986, 2014.

10 Hornberger H, Virtanen S and Boccaccini AR: Biomedical coatings on magnesium alloys - a review. Acta Biomater 8(7): 2442-2455, 2012.

11 Wang J, Tang J, Zhang P, Li Y, Lai Y and Qin L: Surface modification of magnesium alloys developed for bioabsorbable orthopedic implants: A general review. J Biomed Mater Res B Appl Biomater 100(6): 1691-1701, 2012.
12 Yang J, Cui F and Lee IS: Surface modifications of magnesium alloys for biomedical applications. Ann Biomed Eng 39(7): 1857-1871, 2011.

13 Jung O, Smeets R, Porchetta D, Kopp A, Ptock C, Muller U, Heiland M, Schwade M, Behr B, Kroger N, Kluwe L, Hanken $\mathrm{H}$ and Hartjen P: Optimized in vitro procedure for assessing the cytocompatibility of magnesium-based biomaterials. Acta Biomater 23: 354-363, 2015.

14 Jung O, Smeets R, Kopp A, Porchetta D, Hiester P, Heiland M, Friedrich RE, Precht C, Hanken H, Grobe A and Hartjen P: Peogenerated surfaces support attachment and growth of cells in vitro with no additional benefit for micro-roughness in sa (0.24 mum). In Vivo 30(1): 27-33, 2016.

Received November 20, 2017

Revised December 6, 2017

Accepted December 7, 2017 